\title{
Binding of Viral Glycoprotein with Trypsin and Its Relation to Virulency. I. Initial Step of Binding
}

\author{
Takako Shimamoto $^{1)}$, Takuya Shimamoto ${ }^{1)}$, Yoshio Okada1), Nobutoshi Yamada ${ }^{2)}$, Keizo Miyata'), \\ and Yukio Kiho ${ }^{2}$ \\ I'Institute for Molecular and Cellular Biology, Osaka University, Suita, Osaka 565 and ${ }^{2)}$ Central Research \\ Institute, Ishihara Sangyo Kaisha Ltd., Kusatsu, Shiga 525, Japan
}

Key words: viral virulency/trypsin sensitivity

\begin{abstract}
The interaction between surface proteins of some enveloped viruses and trypsin was studied by computer analysis. Prior to the cleavage of the viral protein by trypsin, hydrophobic interaction between them at the vicinity of their active sites may occur. An exposed hydrophobic portion was found there which theoretically could stimulate the interaction. This interaction would be rather non-specific: according to the analysis, trypsin could bind equally well with weakly virulent virus and virulent viruses. Following this interaction, a spe= cific reaction between their active sites would occur. The specificity was found to be related to the virulency of the virus.
\end{abstract}

The virulency of some viruses depends on the sensitivity of their surface $\mathrm{F}$ protein to trypsin. (Proteolytic cleavage at the recognition site of the virus is considered to induce viral infection.) The energy of interaction between these two proteins has been calculated when the active site of trypsin, $170-\mathrm{KDS}$, was positioned at the cleavage recognition site of the viral $F$ protein. The results showed that mainly two kinds of energy, van der Waals energy and electrostatic energy, are decisive for viral virulency (5).

Previously this problem was approached statistically by deviation analysis, which was originally introduced by Kiho (3). It is based on the following principles. i) The frequency of occurrence of each amino acid in a defined sequence with functional significance more or less deviates from uniform distribution, and the amount of deviation (dev) seems to parallel the function of the sequence. ii) Binding of two peptides results in a decrease of their dev values in accordance with the saturation of their binding abilities. Dev analysis is used to deduce the interacting site in proteins. It was found that the active site of trypsin binds to a viral site (dev site) a few amino acids upstream of the cleavage recognition site.

Abbreviations: SV5, simian virus 5; ReSV, respiratory synchitial virus; VISV, Visna virus; NDV (vir), Newcastle disease virus, virulent strain; NDV (avir), avirulent strain of NDV; RSV, Rous sarcoma virus; MMTV, mouse mammary tumor virus; HIV, human immunodeficiency virus type 1; FPV, fowl plague virus; HTLV-1, human T lymphotropic virus; WSN, influenza virus A/WSN/33; PR8, influenza virus PR8; HVJ (Z), Sendai virus, $Z$ strain. The amino acid abbreviations follow standard conventions.
The results further showed that a neighbor to the active site of trypsin also binds to the dev site first, followed by the interaction with the cleavage recognition site and the cleavage reaction (4). Figure 1 shows the dev sites of NDV (vir) for 165-YLE and 170-KDS of trypsin and the cleavage recognition site for 170 -KDS. The details of these sites have already been reported $(4,5)$. In the present study, the interaction energies between trypsin and the dev site of several viral surface proteins were calculated.

\section{MATERIALS AND METHODS}

The interaction energies were calculated as follows. Topological structures of the interacting site of both proteins were constructed using acrophilicity parameters assigned to each amino acid (2). These two structures were then arranged as closely together as possible to visualize the topologically fitted interaction complex. The distances between amino acids in the two structures were determined and three kinds of interaction energy-van der Waals energy $V(V)$, interaction energy between side chain and water $V(S)$, and electrostatic interaction energy $V(E)$-were estimated using the program PEPDENT (5).

Topology in relation to hydrophilicity was investigated using the acrophilicity and hydrophilicity parameter of each amino acid $(1,2)$. Examples of both profiles are shown in Figure 2. There are four main types of extrema: hydrophilic peak (hydrophilicity and acrophilicity are both positive), hydrophobic peak (negative hydrophilicity and positive acrophilicity), hydrophilic depression (positive hydrophilicity and nega- 


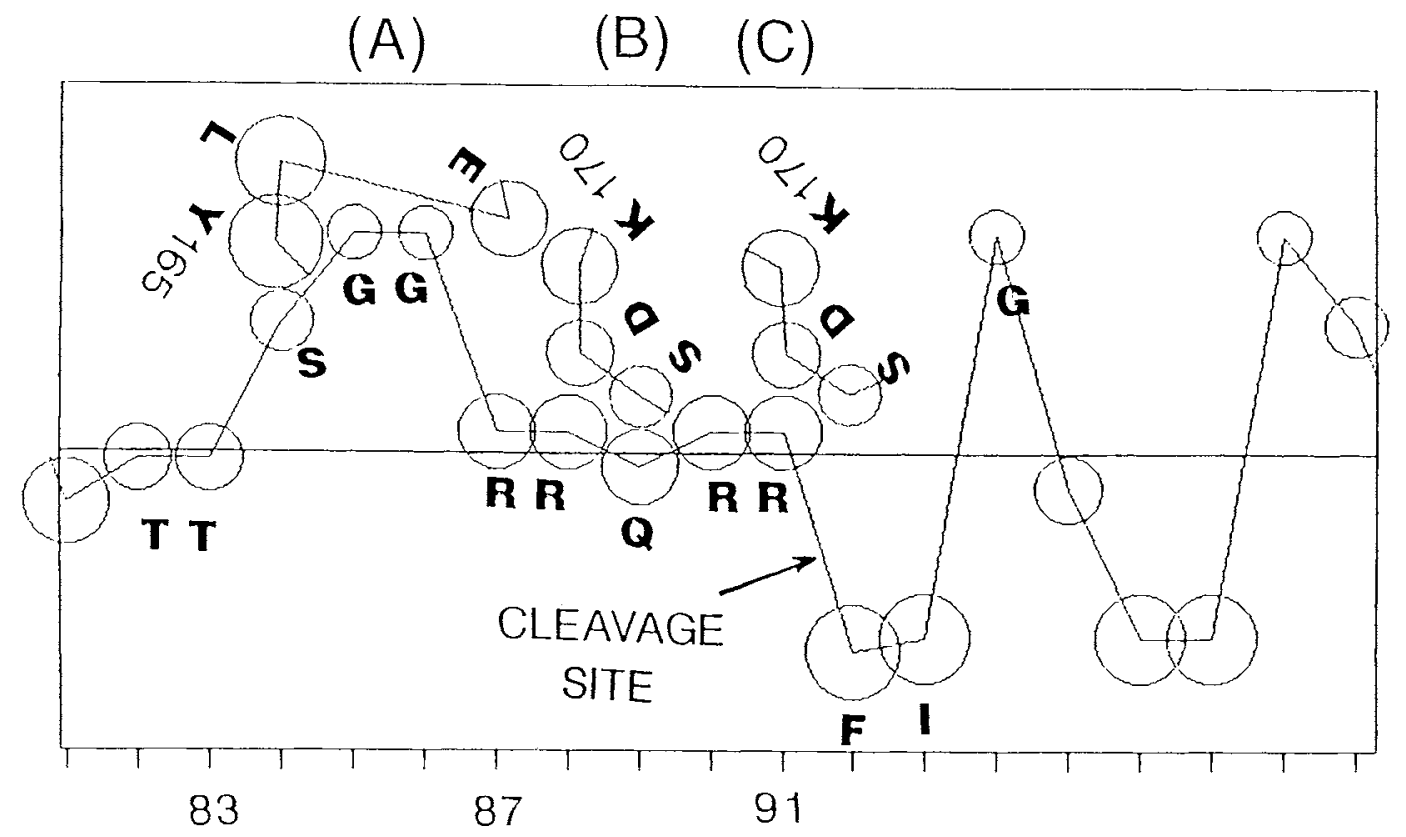

Fig. 1. Interaction between trypsin-YLE or -KDS and NDV(vir) at the dev or cleavage site. The acrophilicity profiles of the dev and cleavage sites of NDV(vir) are shown. The circles represent amino acids. The acrophilicity profiles of two trypsin portions (165-YLE and 170-KDS) were positioned as closely as possible to the viral sites. The distances between the amino acids in the respective molecules were determined on pairs less than $50 \AA$ apart, and V(E), V(V), and V(S) were calculated. (A) Trypsin-YLE at its dev site, (B) trypsin-KDS at its dev site, (C) trypsin-KDS at the cleavage site. The values on the abscissa refer to position in the amino acid sequence of NDV(vir) F protein.

tive acrophilicity), and hydrophobic depression (both hydrophilicity and acrophilicity are negative). We paid the most attention to the second kind, hydrophobic peaks. Using the program "PROPART1" (see Appendix), we selected such regions of the amino acid sequence. The size of a hydrophobic peak, HOPK, was taken as the absolute value of the acrophilicity value times the hydrophilicity value.

\section{RESULTS AND DISCUSSION}

Figure $3 \mathrm{~A}$ shows the energies $\mathrm{V}(\mathrm{E})$ and $\mathrm{V}(\mathrm{S})$ when the active site of trypsin, 170-D, is positioned at the cleavage site of the surface proteins of various viruses (for example, 89-QRRF in the case of NDV(vir) (Fig. 1C)). The values of $\mathrm{V}(\mathrm{E})$ have a positive correlation with the viral virulency (5), which suggests that the electrostatic interaction between the trypsin active site and the cleavage site of the virus is decisive for virulent infection. In contrast, $\mathrm{V}(\mathrm{S})$ is inversely correlated with virulency.

Figure 3B shows the energies $V(E), V(S)$ and $V(V)$ when trypsin $170-\mathrm{KDS}$ is positioned at a dev site of these proteins (for example, 87-RRQR in the case of NDV(vir) (Fig. 1B). In contrast to the case of the cleavage site, the $V(E)$ values are variable and have no relation with the virulency. In some cases, no electrostatic interaction is operative, i.e., $V(E)=0 . V(S)$ also has little correlation with virulency. However, the values are rather uniform among these viruses, which suggests that $170-\mathrm{KDS}$ binds to the dev sites of all viruses rather equally. The pattern for $V(V)$ was intermediate between those of $V(E)$ and $V(S)$.

Figure 3C shows that when trypsin 165-YLE binds to its corresponding dev site of a viral surface protein (for example, 83-TSGGR in the case of NDV(vir) (Fig. 1A)), the values of $V(S)$ and $V(V)$ are uniformly distributed among the viruses considered, and the $V(E)$ values are variable. The above results suggest that trypsin can bind fairly equally to the dev sites of all the viruses studied in this report. V(S) is the controlling factor here, indicating that this process mainly operates by hydrophobic association. The binding is followed by the interaction with the cleavage site, in which the electrostatic force is the main factor on which viral virulency depends. Thus the first step is rather non-specific, but the second step is specific. These features coincide with the natures of $V(S)$ and $V(E)$, which control these processes. By the first hydrophobic interaction, the dielectric constant of the surrounding medium will decrease, which in turn stimulates the ionic interaction, resulting in the transfer of trypsin from the dev site to the cleavage site.

The first step can occur easily if the hydrophobic portion of the virus concerned is exposed on its surface (hydrophobic peak). Energetically, exposure of a hydro- 


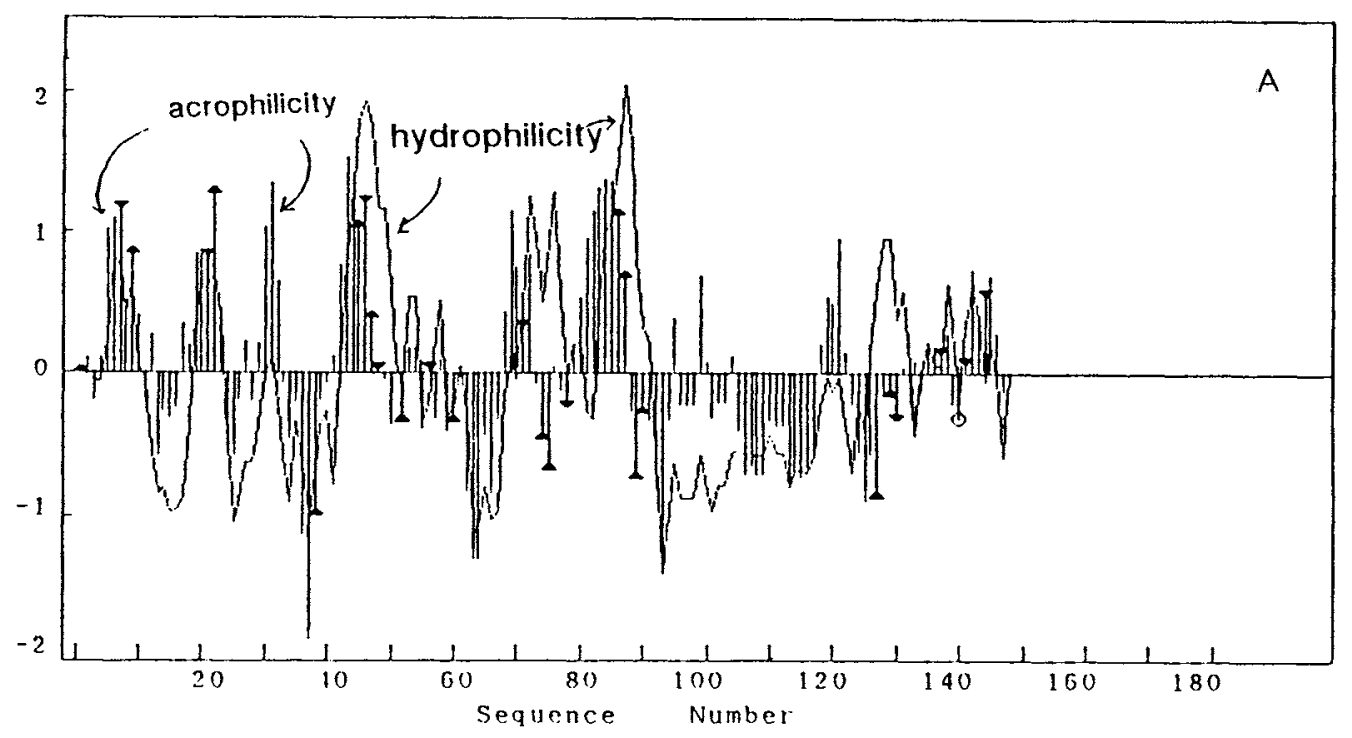

A Hydrophilicity and Acrophilicity

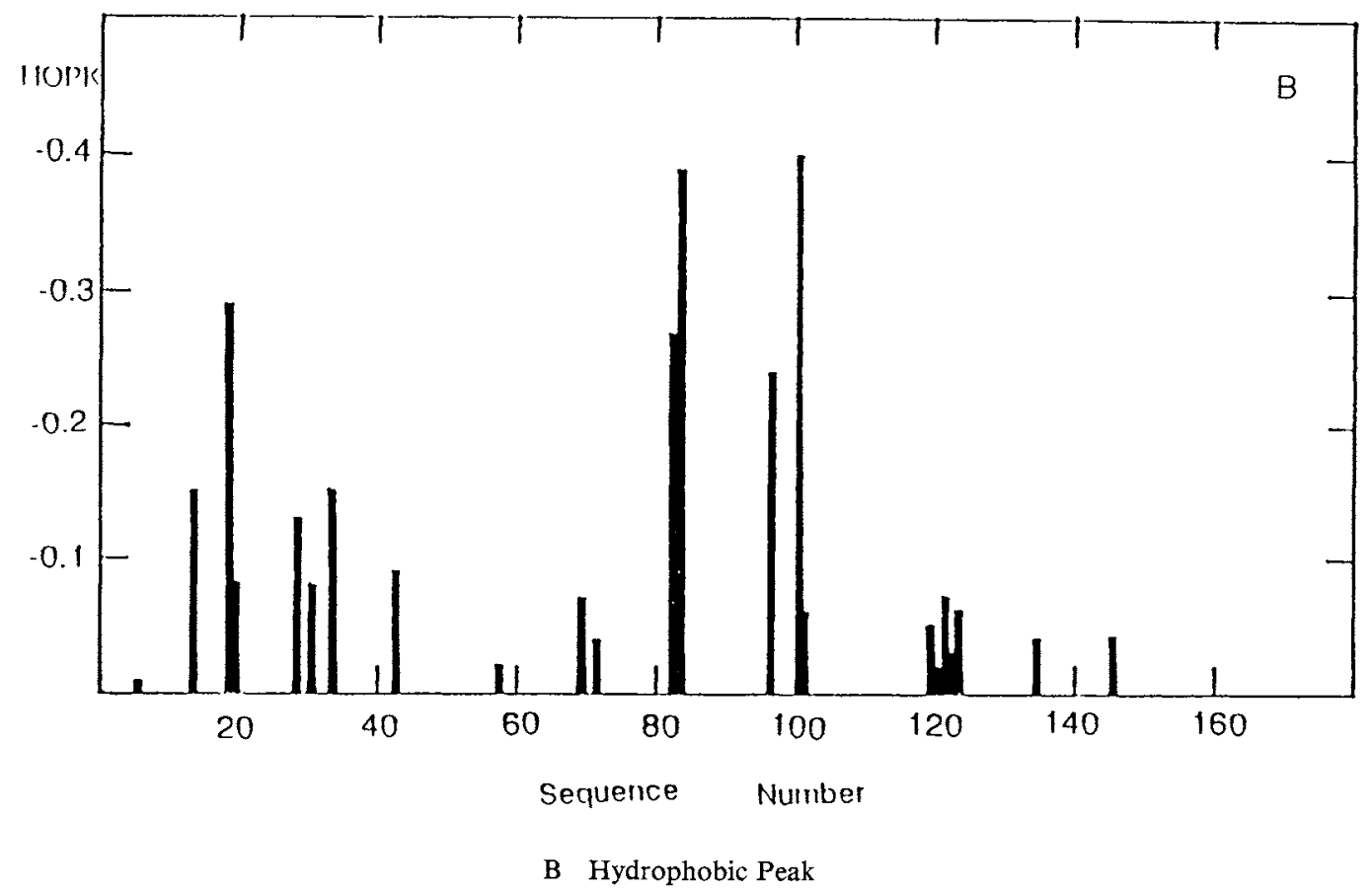

Fig. 2. (A) Hydrophilicity (shown by the curve) and acrophilicity (shown by the vertical bars) profiles of F protein of NDV(vir). The hydrophilicity or acrophilicity values (ordinate) are averaged for six amino acids consecutively along the sequence (abscissa). The cleavage site and dev sites are in the region between residues 80 and 90 . (B) The hydrophobic peaks found in the same region as above are shown. The ordinate indicates the HOPK values.

phobic portion in aqueous medium is rather rare. Although rare, hydrophobic sites on the surface could easily bind with another hydrophobic sites. Figure 2A shows the hydrophilicity and acrophilicity profiles of the portion of F-protein of NDV(vir) in which two dev sites and the cleavage site occur: in the region between residues 80 and 90 . The hydrophobic peaks are shown in Figure 2B. The most notable HOPKs were found at 82-TT-83, whose values were 0.2692 and 0.3833 at $82-\mathrm{T}$ and $83-\mathrm{T}$, respectively. These residues are exposed around the dev sites for trypsin binding. Two other important HOPKs were found at $18-\mathrm{VV}-19$ and at $100-$ 


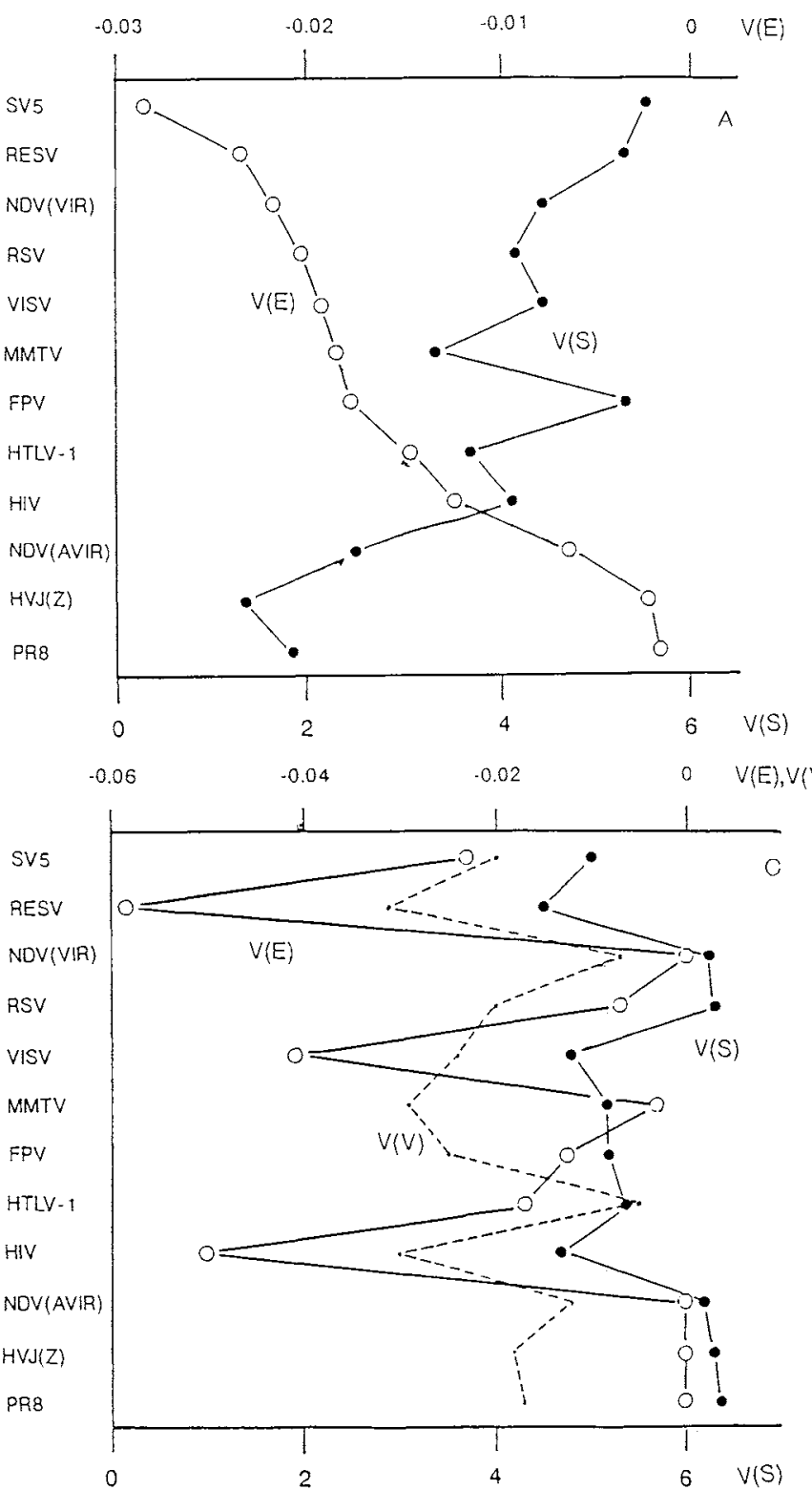

VA-101. The other peaks were small and scattered. Furthermore, a hydrophobic peak was also found in trypsin close to the active site, at 165 -YL-166. Its HOPK values were 0.0667 and 0.1528 at $165-\mathrm{Y}$ and $166-\mathrm{L}$, respectively. The interaction of these hydrophobic peaks would faciliate the binding of trypsin 165-YLE to the viral dev site, and this step is most affected by V(S).

Hydrophobic peaks near the binding site (dev site) were also found in other cases, including various $F$ proteins of paramyxoviruses, as shown in Table I. This raises the possibility that the above discussion can be generalized. In the binding reaction of biological macromolecules, it is frequently said that the first interaction is ionic. For example, at the initial stage of viral infec-

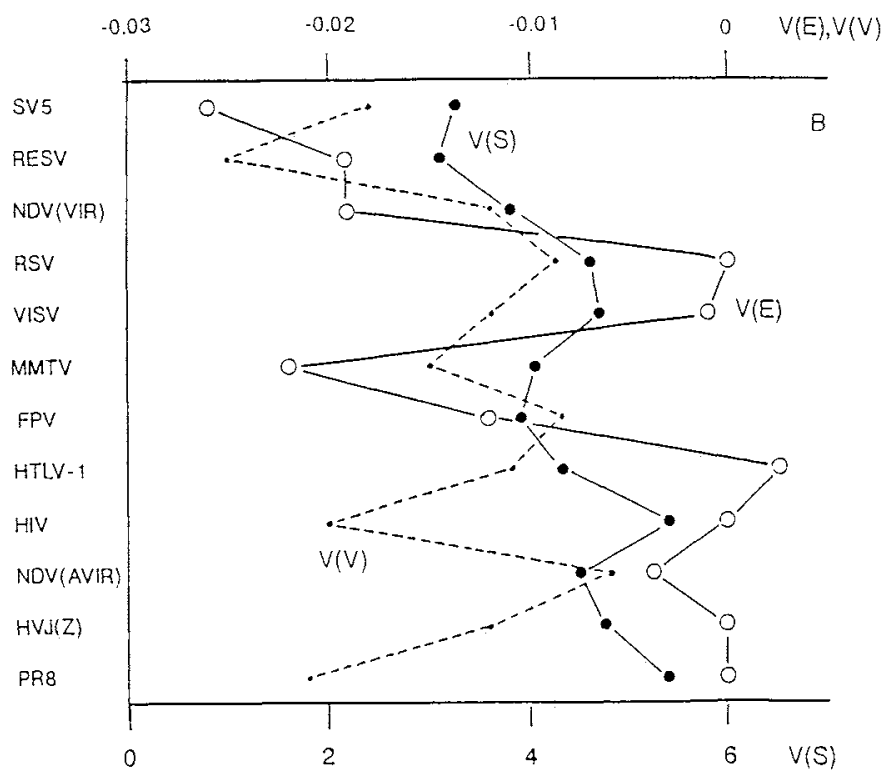

Fig. 3. Interaction energies of enveloped viruses. (A) Tryp$\sin 170$-KDS interacting with the cleavage site. (B) Trypsin 170KDS interacting with the corresponding dev site. (C) Trypsin 165-YLE interacting with the corresponding dev site.

tion, the adsorbed virus can be released by salt at high concentration. (After adsorption, the virus penetrates the membrane and enters the cell, where the hydrophobic interaction is operative and the infected virus cannot released by high salt.) In view of the large value of the dielectric constant of water, it is difficult to consider simple ionic interaction as the first step of viral adsorption. Binding other than or in addition to ionic interaction, such as the binding of trypsin to the viruses described above, should be considered. 
Table I. Distances (IN terms of amino acids) BetWeEn THE BINDING SITE OF TRYPSIN-YLE (A DEV SITE),

THE HYDROPHOBIC PEAK, AND THE CLEAVAGE SITE (2) IN VARIOUS VIRAL PROTEINS.

\begin{tabular}{lcc}
\multicolumn{1}{c}{$\begin{array}{c}\text { hydrophobic } \\
\text { veak }\end{array}$} & \multicolumn{2}{c}{$\begin{array}{c}\text { dev site for } \cdots \\
\text { trypsin-YLE }\end{array}$} \\
\hline virus & $c$ & $\mathrm{a}$ \\
\hline SV5 & $8-11$ & $2-5$ \\
ReSV & 7 & 4 \\
NDV (vir) & $1-2$ & 8 \\
RSV & -1 & 7 \\
VISV & 16 & $5(3)$ \\
MMTV & 16 & 2 \\
FPV & 13 & 3 \\
HTLV-1 & 5 & 2 \\
HIV & $5-8$ & $7-10$ \\
NDV (avir) & -2 & 9 \\
HVJ (Z) & -4 & $8(4)$ \\
PR8 & -1 & 5 \\
\hline
\end{tabular}

Acknowledgment. We are grateful to Dr. A. Ubasawa for writing the computer program and for stimulating discussions.

\section{REFERENCES}

1. Equchi, Y. and Asou, Y. (1985). BASIC in Biochemistry (Japanese) Kyoritsu Publ., Tokyo. pp 16.

2. Hopp, T.P. (1985). Prediction of protein surfaces and interaction sites from amino acid sequences. In Synthetic Peptides in Biology and Medicines (K. Alitaro, P. Partanen and A. Vaheri, eds.). Elsevia Sci. Publ., pp 3-12.

3. Kıно, Y. (1988). Regional variation and function of nucleotide and amino acid sequence. Cell Struct. Funct., 13: 387-405.

4. Kino, Y., Mirata, K., and OKada, Y. (1989). Prediction of the virulencies of some enveloped viruses from the structure of the cleavage recognition site of viral glycoprotein essential for infectivity. II. Deviation analysis. Cell Struct. Funct., 14: 721730.

5. Okada, Y., Shima, Y., Shimamoto, T., Kusaka, N. and Kiho, Y. (1989). Prediction of the virulencies of some enveloped viruses from the structure of the cleavage recognition site of viral glycoprotein essential for infectivity. I. Calculation of interaction energy. Cell Struct. Funct., 14: 707-719.

(Received for publication, September 25, 1990 and in revised form, November 21, 1990) 


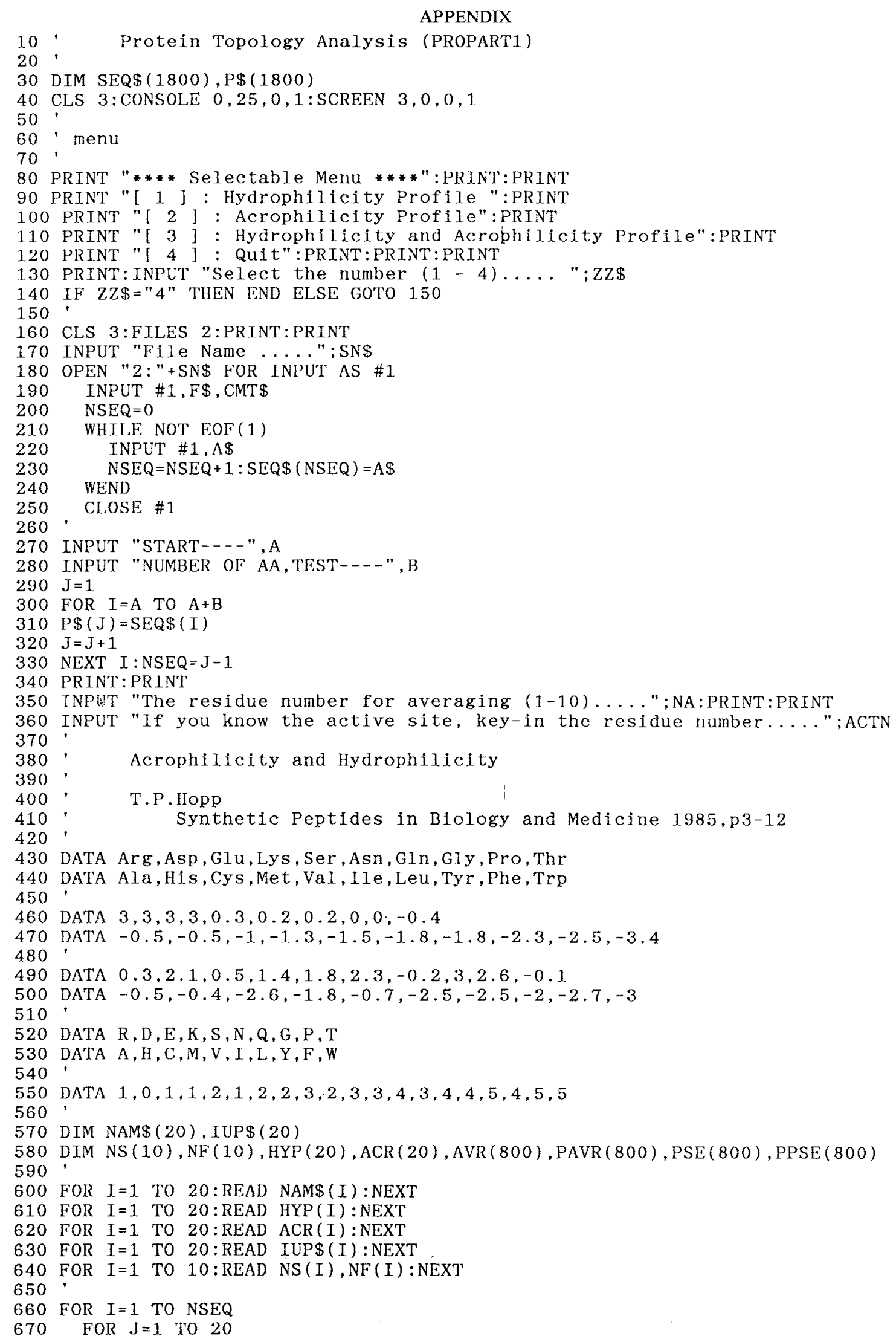


Binding of Virus with Trypsin

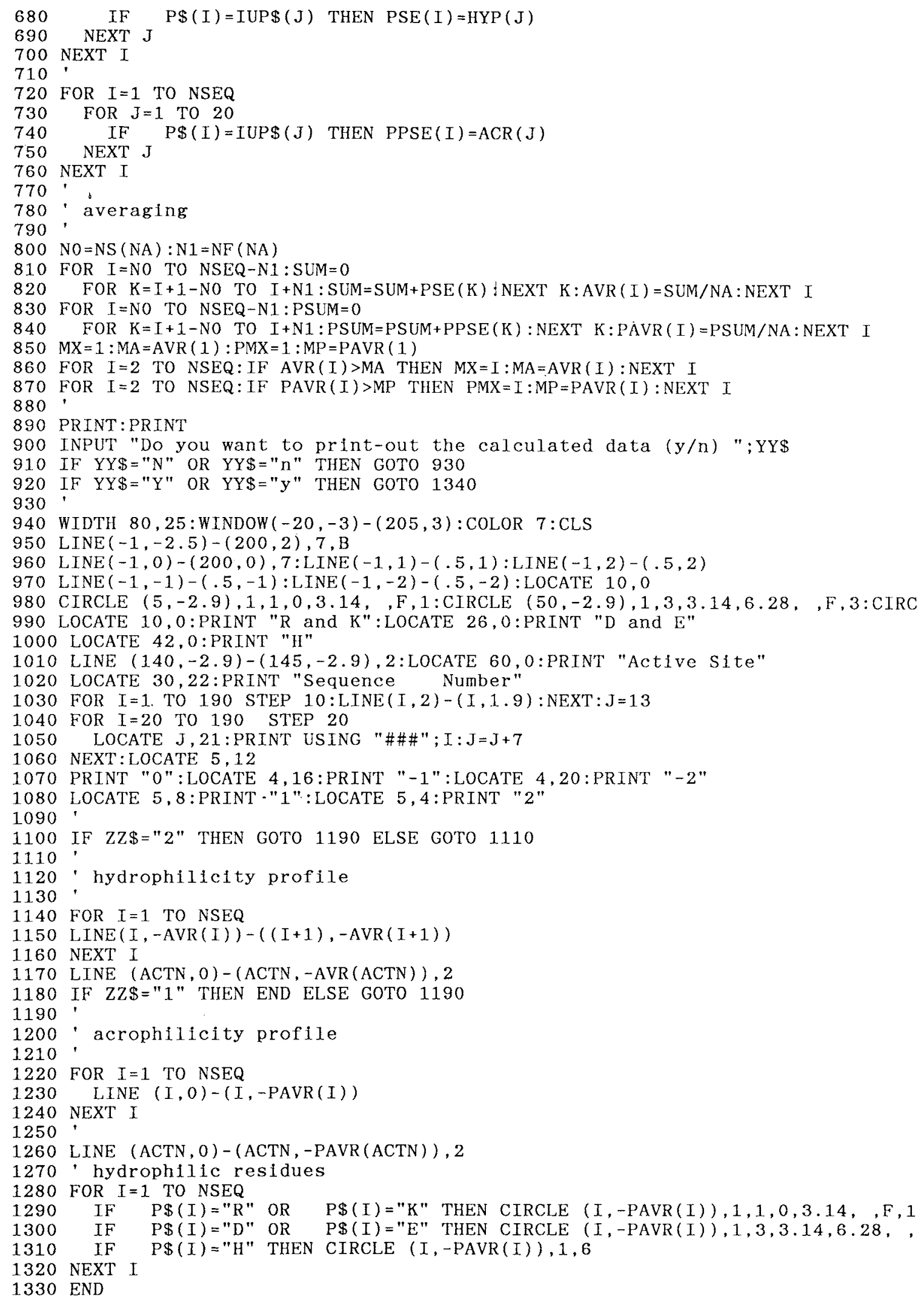


1340 ' print-out data

1350

1360 LPRINT CHR\$ (27);" !";"< " CMTS;" > :LPRINT:LPRINT

1370 LPRINT CHR\$(27); CHR\$(\&H22);

1380 LPRINT "Averaging group length =";NA:LPRINT

1390 LPRINT " [Sequence] Hyd Acr
1400 LPRINT

1410 FOR $I=1$ TO NSEQ

1420 FOR $K=1$ TO 20

$1430 \quad$ IF $\quad P \$(I)=I U P \$(K) \quad T H E N \quad A A \$=N A M \$(K)$

1440 NEXT K

1.450 LPRINT USING "\#\#\#\&\& \& \& ";I, P\$(I), $\wedge \Lambda \$ ;$

1460 LPRINT USING "\#\#.\#\#\#\#.\#\#"PSE(I), PPSE (I);

1470 . IF $I<N O$ THEN LPRINT:GOTO 1500

1480 IF I>NSEQ-N1 THEN LPRINT:GOTO 1500

1490 LPRINT SPC (10); USING "\#\#.\#\#\#\#\#\#.\#\#\# 1500 NEXT

1510 GOTO 930 